\title{
Bereaved by Suicide
}

\section{Said Shahtahmasebi ${ }^{1,2,3 *}$ and Caroll Aupouri-Mclean ${ }^{1}$}

${ }^{1}$ Centre for Health and Social Practice, WINTEC, Hamilton, New Zealand

${ }^{2}$ Director of Research, The Good Life Research Centre Trust, Rangiora, New Zealand

${ }^{3}$ Voluntary Faculty, Division of Adolescent Medicine, University of Kentucky, USA

\begin{abstract}
Parents and family members are often treated as experimental or observational units from whom information can be gathered and inferences made about suicide survivors (family of a suicide case). Collecting data in this way limits the knowledge about suicide survivors' needs and how they can contribute to the suicide debate for the development of a suicide policy. It is not helpful to claim mental illness as the cause of suicide when the suicide rate decreases, and then claim that suicide is a very complex health outcome caused by many factors when the suicide rate increases. In this discussion paper, we argue that a suicide prevention model that places mental illness at the centre of the suicide debate only leads to a policy of 'more of the same', and will have little effect on suicide prevention and care support post-suicide (for suicide survivors). Furthermore, we argue that the contribution that suicide survivors can make to understanding suicide should not be ignored.
\end{abstract}

\section{Introduction}

Suicide has been investigated from the perspectives of many different disciplines including medicine, psychology, sociology, spirituality and religiosity, economic and environment. Suicide can be prevented but its cause(s) is complex which cannot be explained by one discipline or a single model. Suicide research is particularly subjective because the key people who could potentially provide insights into their process of decision making are no longer alive. Most suicide research has relied on information gathered from family and friends (suicide survivors) and from medical records after the event e.g. [1-3]. The problem with suicide research is that information taken from family and friends after the event of suicide is highly biased, and, up to three-quarters of suicide cases do not come into contact with psychiatric services [4-6]. Untimely death, due to suicide or accidents has multiple victims; individuals who lose their lives to suicide or accident, family and friends bereaved by suicide. The victims who are bereaved by suicide such as family are often known as survived by a suicide or suicide survivors [7]. The literature suggests heterogeneity within this group e.g. suicide survivors may be different to those bereaved by accident or homicide [8]. As a group, suicide survivors' health outcomes and mental wellbeing has been considered to be a higher risk than the general population [911]. Suicide survivors have also been studied as victims, compared with other bereavement groups and identified as a risk group for health and social needs assessments $[8,10-13]$. Such studies are often driven by the authorities' or researchers' theories or hypotheses that will dictate questions for suicide survivors to answer. In this discussion paper we discuss that although, such a top-down approach has its advantages it will limit access to rich data sources: the survivors.

\section{Background}

Suicide is probably the most researched public health topic. However, despite the volume of literature, there is confusion about the causes of suicide on which, at least in part, suicide prevention policies are based [5,6,14-17]. Our knowledge of suicide is, at best, based on scant scientific evidence [18-20].

The literature on suicide, e.g. [21], suggests a large number of variables from depression to trauma, bereavement, unemployment, marriage break up, poor health, drug and alcohol abuse, poor nutrition, childhood events and so on may cause suicide. A pragmatic translation of the literature is that the general population is at risk of suicide. This interpretation actually makes sense and reinforces that suicide cannot be explained by one discipline or a model. Suicide is a process of decision making. Suicide prevention policy development should allow a multidisciplinary approach to include input from members of the public.

However, suicide prevention is firmly based on a medical model that seeks a cause (mental illness) leading to an effect (suicide). Such a model has not been successful for a number of reasons. First, if mental illness is the cause of suicide then on the one hand we would expect a much higher suicide rate in the population with mental illness and on the other hand in the general population. Second, we would expect a reduction in the suicide rate due to medical interventions and treatment. Third, as previous studies have shown suicide rates appear to have a cyclic trend $[14,22]$ which means that medical interventions have little or no impact, because despite these interventions the rates will change course over time. Fourth, there have been cases of people who had been treated for mental illness due to suicide attempts or ideation but completed suicide following medical treatment and discharge.

One of the consequences of the medical model is the expectation of an answer to the question 'why'. But, suicide is the outcome of a decision process and a clinical 'cause' does not address issues that are relevant to the process which culminates in the act of suicide $[14,17,23]$. For example, there is a time delay between the incidence of a negative life event and the act of suicide. Very little is understood about the decision making process during this time period. We are still vague about the dynamics of the decision making process that leads an individual to commit suicide while others with similar characteristics do not. What is fairly clear is the impact of suicide on those left behind. For example, in addition to the complexities of dealing with losing a

${ }^{*}$ Corresponding author: Said Shahtahmasebi, Centre for Health and Socia Practice, WINTEC, Hamilton, New Zealand, E-mail: Said.Shahtahmasebi@ wintec.ac.nz

Received November 02, 2011; Accepted December 07, 2011; Published December 09, 2011

Citation: Shahtahmasebi S, Aupouri-Mclean C (2011) Bereaved by Suicide. Primary Health Care: Open Access 1:101. doi:10.4172/2167-1079.1000101

Copyright: (c) 2011 Shahtahmasebi S. This is an open-access article distributed under the terms of the Creative Commons Attribution License, which permits unrestricted use, distribution, and reproduction in any medium, provided the original author and source are credited. 
loved one to suicide, the literature suggest differentials in the nature of grief and mourning and healing process in suicide survivors compared with bereavement due to other causes e.g. accident $[8,11,24,25]$.

As human beings, it is natural to sympathise with the tragedy of loss to suicide of another human being. However, it is not easy to comprehend the dynamics of the process of coping with the aftermath of suicide of a loved one. The process outcome may not necessarily be acceptance of, or, coming to terms with the event. It is not correct to use the term healing process because suicide often leaves an emotionally painful scar. The size of the scar and the magnitude of the pain are governed by individual characteristics and family and social processes. For example, the stigma of suicide and mental health [12] can lead to social isolation and therefore adversely affect suicide survivors' health and social outcomes.

It is not surprising that the needs of suicide survivors and possible interventions have been receiving some attention in the literature $[8,11,13,24,26-28]$. What is lacking from a discussion of survivors' needs is a thorough debate on how the socio-political processes impact suicide survivors, and more importantly suicide itself. To develop preventional and care support polices based on evidence, suicide must be understood as a dynamic process. In other words, the process of decision making is interconnected and influenced by other human behaviour processes such as cultural, socio-economic, sociopolitical, and socio-environmental. Available suicide databases do not lend themselves to study the reasons as to why over two-third of the suicide cases do not come into contact with mental health services [46]. And only for a fraction those suicide cases who have a psychiatric record depression is recorded as the main diagnosis [5]. Substance abuse, drug/alcohol dependence, schizophrenia also feature in a small minority of records [5]. In some countries, such as New Zealand, the official line of keeping suicide out of the public domain has helped suicide to maintain its taboo status. Ironically, the policy of secrecy is being enforced at a time where resources are being spent to make mental illness more mainstream in order to remove its taboo status. So there is confusion in the public mindset which may exacerbate the adverse effects of a suicide.

The approach to suicide prevention, through a policy of secrecy has been to effectively silence the survivors. In other words, our actions treat survivors as experimental units, even in cases where the research is qualitative, the discussion and information gathering is directed and guided by the researchers' theories and agenda.

\section{Discussion}

The literature on suicide survivors is relatively small. A search in Google Scholar generated 70,000 hits using the search term 'survivors of suicide' while, a similar search using the term 'suicide' alone generated 1,250,000 hits. Unlike suicide research, the main informants in the suicide survivors' research are alive and may be willing to provide information about themselves. However, studies of survivors have tended to describe complex emotional and personal grief following the act of suicide by a family member. For most families the loss of a loved one to suicide is sudden and without prior warning and for which there is no explanation. It is plausible to assume that over and above health needs, for most survivors a lack of explanation and a lack of information on suicide, together with social perceptions and attitudes to suicide may exacerbate coping with loss to suicide $[29,30]$. However, some of these social barriers may be a direct result of social and health policies on suicide. In New Zealand, the policies are vague (e.g. see
[16]) and there is a lack of public debate in terms of increasing access to information, in particular for the youth, to prevent future suicides. And, anyone with depression or suicidal ideation, or those who have concerns about someone are strongly advised to contact mental health services. This approach can cause frustration with the survivors and can potentially exacerbate the suicide survivor's process of coping. A social code of secrecy and replacing 'suicide' with 'undetermined injury' in order to prevent 'copycat suicide' is absurd and isolates survivors in their grief.

Theories of grief developed in the 1960s have been influential in understanding bereavement. Kubler-Ross [31] describes a five-stage model of grief and bereavement that includes denial, anger, bargaining, depression and acceptance. Extreme grief reactions include depression, anxiety and post-traumatic stress disorder which can influence the length of the bereavement process, making it longer with a slower recovery period $[8,11,12,32,33]$. However, human behaviour such as grieving is a dynamic process, and, often attempts to model human behaviour fails because most models fail to account for temporal changes and dynamics in behaviour [34]. For example, some high school and community based studies suggest that grief due to suicide is too complex to fit the Kubler-Ross model $[35,36]$.

The experiences and needs of families bereaved by suicide have been under-represented in the literature and families have been described as the forgotten people [37]. A larger proportion of the forgotten people are often the indigenous and minority groups. For example, whilst there has been increasing recognition of the need to better understand the responses of those who are bereaved by suicide, literature on Maori whanau (immediate and extended family) bereaved by suicide is virtually non-existent. Maori have been less visible in the research for a number of reasons. Firstly, an abundance of studies, especially the earlier studies, focussed more on Western experiences of suicide. Secondly, the subject of 'suicide' has been taboo in Maori society. This is acknowledged in the New Zealand Youth Suicide Prevention Strategy which includes, as one of the five major goals of the strategy, "the provision of effective support to those who are bereaved or affected by a suicide" [21]. The same document gives prominent to mental illness as the cause of suicide but argues that suicide is a complex matter with social, education, economic, environmental and life experiences as contributing factors. Such a political approach clearly tips the balance of resource allocation overwhelmingly towards mental health and psychiatric units. Thus, the link with mental illness is well maintained, which does very little in attenuating the taboo status. The question is then whether or not maintaining a link between mental illness and suicide is the reason for a lack of service uptake by those who really need it? The alternative question is whether a lack of appropriateness of mental health services to suicide is the reason for a lack of service uptake?.

Indeed, anecdotal evidence in the literature and personal observations suggest a great deal of anger is felt by suicide survivors $[11,12,33]$. The circumstances and other social and environmental factors, such as social stigma and a lack of answers and information, add complexities to the nature of the anger. It is safe to assume that it may take a very long time to deal with a complex anger in particular where those responsible for their actions, i.e. the suicide cases cannot be held accountable. The anger may be directed towards the rest of the community which affects relationships within the family and with friends and colleagues, and may lead to isolation. The main question here is whether our support services are equipped to deal with this 
complex anger and support individuals. However, not enough is known about suicide survivors and the nature of their anger. Is it reasonable to assume that time will help them to come to grips with this type of anger regardless of any support? Nevertheless, anger along with other individual and social issues must be treated as part of suicide which is a dynamic human behaviour process.

It is plausible that the anger reportedly felt by suicide survivors may have its roots in our attempts to find a cause for suicide. A medical model seeks to quantify and relate identified symptoms to an outcome that may then lead to a diagnosis or a series of diagnoses for which a course of treatment is prescribed. The patient is monitored and the course of treatment may be modified if symptoms persist. Suicide does not fit into this model. The subject is no longer alive to provide the crucial information needed to assess their health status. Over twothirds of suicide cases do not come into contact with the authorities. Our knowledge of suicide and suicide cases is based on those who have had some contact with the authorities. It is reasonable to assume that our understanding of suicide is therefore speculative at best. In the absence of a healthy public debate such speculations become set in the public mindset as facts about suicide. It is also reasonable to assume that speculating about suicide is not without its consequences for society as a whole. The current guidelines encourage the public to refer those who appear depressed or suicidal to the mental health services in the first instance. Over two-thirds of suicide cases do not come into contact with mental health services and anecdotal evidence suggests that those who did seek help still lost their loved one to suicide $[5,6,14]$. On the other hand, knowing that they might be referred to mental health services, a person with suicidal ideation may hesitate in confiding to a relative or friend. Furthermore, when no cause can be found, speculations and the lack of information may impact the suicide survivors well beyond the loss of a loved one. The literature suggests adverse mental and emotional effects on suicide survivors (e.g. parents, siblings, relatives and friends) following a suicide $[8,10-12,24,27,29,33,38]$. Suicide survivors choose to deal with such a traumatic experience despite the medical model. Nevertheless, speculating mental illness and negative life events or a negative experience as causes of suicide would encourage survivors to look for such causes by association. Although this is a natural reaction, survivors continue a life of self-blame for not identifying their loved one's mental illness that may not have even existed. Anecdotal evidence suggests that even in cases where problems were identified and help was sought, survivors still continue to blame themselves for not doing enough; perhaps if they had sought a second opinion, or alternative treatment. Thus, speculation may adversely impact human behaviour which in turn has implications for service development and service provision pre- and post suicide. Speculations about the causes of suicide may inadvertently shift the responsibility of a suicide to the survivors by implying unnoticed mental illness, childhood trauma and abuse and other events that the survivors would have been in a position to prevent. Such an approach gives rise to a conflict between social beliefs and attitudes to suicide, social behaviour, social justice and individual's rights. For example, why should suicide survivors be, implicitly or explicitly, blamed for the suicide of a loved one? Mental illness is still a social taboo. It is, therefore, plausible that this socially accepted causal link between mental illness and suicide may lead to social isolation of the survivors.

It is all very well for suicide research to recommend preventional policies, but, without a clear insight and a good understanding of suicide, preventional policies will be potentially ineffective. For example, New Zealand Government's policy advocates programmes that emphasise individual resiliency to suicide [21]. In other words, resiliency helps to prevent suicide as well as help suicide survivors to cope with their loss. The concept 'resilience' has been defined as a psychological trait, with the ability to problem solve and having a higher sense of self and being able to cope with life events $[39,40]$. The ability to manipulate personality traits to enable problem solving does not necessarily equate to higher levels of resiliency whilst suicide remains a viable solution to life's problems. While problem solving can be taught, resilience or developing a sense of self-belief, solving a problem and a desire to live may be more individual-specific. On the other hand, if personality traits can be manipulated to promote resiliency, by the same token they can also be manipulated in the opposite direction and weakened by the same social parameters and environmental, political and economic forces that gave rise to a desire to strengthen resilience.

Most studies of suicide survivors ignore the multi-dimensionality of suicide and appear to follow a top-down approach [15] and are mainly concerned with identifying health and emotional issues of survivors in relation to health care service provision, e.g. $[8,12,26,29,41,42]$. In other words, we the researchers and policy makers have been readily making assumptions about suicide and suicide survivors. Over the last decade proponents of the medical model concentrated on tackling depression as one of the main causes of suicide. The strategy included depression awareness campaigns. From these investments we would have expected a reduction in suicide rates, or at least no change, but what we did not expect was the result that suicide is on the increase and we need new solutions. If the search for new solutions follows the current trend then it would only mean one thing: more of the same! In 2011, in the face of rising suicide rates, the New Zealand's Chief Coroner stated "These statistics clearly show that what we have done in the past is not bringing the toll down so we must look for new solutions". http://www.stuff. co.nz/national/health/5513217/Suicide-numbers-drop-after-quake. Clearly, new solutions do not come from the same model and modellers but from an inclusive grassroots approach.

In 2010, the Waikato Institute of Technology (Wintec) organised and hosted youth suicide prevention workshops in the Waikato Region. The workshops were presented by the Chairman of the 'stop youth suicide campaign' (www.stopyouthsuicide.com) in Kentucky, USA. These workshops received considerable interest from the public at grassroots, including community, health and social workers, police, those concerned about what suicide is doing to their community, those who had lost a loved one to suicide, and teachers. Ironically, no academics, no one from the medical profession, and no politicians attended the workshops. Some community groups attended the workshops in spite of being advised not to attend by their suicide prevention peers. These are indications that suicide is highly politicised which prevents us understanding suicide and its prevention. Until the public demand a change in suicide prevention policy there will be more of the same: the same solutions, the same services, the same decision makers, the same evidence provided by the same group of advisers/ researchers. It is not surprising that there is mistrust between the public and care providers, in particular suicide survivors (family and friends). Current estimates suggest over two-thirds of suicide cases do not come into contact with mental health services/psychiatric units [4-6].

One of the interesting issues that arose from the workshops was the will of suicide survivors to survive, and that they would like to be heard, and are desperate to contribute to the debate. They would like to make the general public aware and potentially prevent suicide in other families. However, the New Zealand's Associate Minister 
for Health caused anger and concern amongst suicide survivors by leaving them out of his review of suicide (http://www.stuff.co.nz/thepress/news/4550480/Anger-over-missing-voice). In other words, the confidence and resilience of suicide survivors is further undermined because they are not considered important enough by planners to contribute or inform the process of policy formation. This is an artefact of a top-down approach to decision-making which leaves the authorities ignorant of the resulting 'more of the same' policies and services $[15,16]$.

Clearly, suicide survivors need a system of care support available to them at micro- and macro-level. The characteristics and structure of family and friends, and the characteristics of society/community and social environment will influence the development of care support at micro- and macro-level. How important are family and social characteristics in lowering suicide rates? Holistically viewed, it is important to consider the impact of social, health and economic policies on family and community characteristics as well as the changing social expectations. Of course, social expectations are a multi-way influence: an individual's expectations of society and vice versa, family expectations of society and vice versa. What family and societal characteristics contribute to resilience? Are resilient families more protected against suicide? And by the same token are resilient societies/communities more protective of family members against suicide?

The resilience of suicide survivors surviving within a community should not be confused with the social resilience of that community. There are communities with higher rates of suicide than others and yet survivors show a strong will to survive and be supportive of others. How resilient a community is may be based on its preventative measures that the whole community might subscribe into. The literature is vague on the nature of effects, if any, on suicide survivors from the provision of supportive care after the suicide, e.g. does post suicide support services help a survivor's resolve to cope? The questions that will need to be sought from suicide survivors is whether care packages reach them in time and whether the support packages are equipped to deal with their particular needs? How do survivors cope and compensate for unnoticed and undiagnosed mental illness as the cause of death of their loved one? And what about the multiple effect of social views of suicide and mental illness on the survivors? Therefore, it is reasonable to ask the question "how and with what mechanism do the next of kin of suicide cases survive the massive and sudden impact of bereavement?"

\section{Conclusion}

Over the last decade, authorities in New Zealand have been running a massive programme of mental illness and depression awareness as part of a suicide prevention campaign. This programme coincided with the downturn in the suicide cycle. Naturally, authorities who have invested resources in this programme readily made the connection with suicide and promoted it. However, current statistics suggest that the downturn cycle ended a couple of years ago. That is the reason for the Chief Coroner questioning the current approaches to suicide prevention calling for new solutions as stated earlier.

Any new solutions for suicide prevention must be conducive to change our current perceptions of suicide. In other words, new solutions operating within the old or the existing modelling framework are constrained to deliver the old model outcomes. An examination of policy and strategy documents on mental health suggests that promises of new solutions, new approaches and new directions appear to translate into policy actions that lead to 'more of the same' [16]. There is little or no value in glossing over existing problematic issues by sub-branching with socio-economic and individual characteristics such as resilience as new solutions. The overhaul, as part of a new solution must be inclusive at grassroots level, to give a greater voice to suicide survivors.

Suicide survivors have a story to tell that can increase our insight into suicide but a top-down approach of studying this group shifts the emphasis away from suicide. The literature claims suicide survivors grieve differently and that they suffer higher levels of guilt, blame, and responsibility, and greater feelings of rejection and abandonment; stronger feelings of stigmatization and social isolation and a particular impact on family systems including family interaction and communication $[8,11,12,33,43]$. Anecdotal evidence suggests that due to a lack of appropriate suicide prevention programmes and postsuicide support, survivors are not empowered to grieve the way they want or would like to. Suicide survivors are alive and only they can tell their story and contribute to this debate.

\section{References}

1. Agerbo E, Nordentoft M, Mortensen PB (2002) Familial, psychiatric, and socioeconomic risk factors for suicide in young peopl: Nested case-control study. BMJ International 325: 74.

2. Beautrais AL, Joyce PR, Mulder RT (1994) The Canterbury suicide project: Aims, overview and progress. Community Mental Health in New Zealand 8 : 32-39.

3. Appleby $L$ (1992) Suicide in psychiatric patients: risk and prevention. British Journal of psychiatry 161: 749-758.

4. Hamdi E, Price S, Qassem T, Amin Y, Jones D (2008) Suicides not in contact with mental health services: Risk indicators and determinants of referral. J Ment Health 17: 398-409.

5. Shahtahmasebi S (2003) Suicides by mentally ill people. ScientificWorldJournal 3: 684-693.

6. Shahtahmasebi S (2005) Suicides in New Zealand. ScientificWorldJournal 5 527-534.

7. Andriessen K (2009) Can postvention be prevention?. Crisis 30: 43-47.

8. Bailley SE, Kral MJ, Dunham K (1999) Survivors of suicide do grieve differently: Empirical support for a common sense proposition. Suicide and LifeThreatening Behavior 29: 256-271.

9. Farberow NL, Gallagher DE, Gilewski MJ, Thompson LW (1987) An examination of the early impact of bereavement on psychological distress in survivors of suicide. The Gerontologist 27: 592-598.

10. Grad OT (1996) Suicide: how to survive as a survivor?. Crisis 17: 136-42.

11. Hawton K, Simkin S (2003) Helping people bereaved by suicide. BMJ 327: 177

12. Cvinar JG (2005) Do suicide survivors suffer social stigma: A review of the literature. Perspectives in Psychiatric Care 41: 14-21.

13. Demi AS (1984) Social adjustment of widows after a sudden death: Suicide and non-suicide survivors compared. Death Education 8: 91-111.

14. Shahtahmasebi S (2008) Suicide Research and Adolescent Suicide Trends in New Zealand. ScientificWorldJournal 8: 287-302.

15. Shahtahmasebi S (2009) Suicide prevention in a top-down society. In Suicide in the military 121-136.

16. Shahtahmasebi S (2012) A Review and critique of Mental Health Policy Development. Int J Child Health Hum Dev 5: 3

17. Shahtahmasebi S, Shahtahmasebi R (2010) A Holistic View of Suicide: Socia Change and Education and Training. Journal of Alternative Medicine Research 2: $115-128$.

18. Cutcliffe JR (2003) Research endeavours into suicide: a need to shift the emphasis. Br J Nurs 12: 92-99. 
19. De Leo D (2002) Why are we not getting any closer to preventing suicide?. Br J Psychiatry 181: 372-374.

20. Institute of Medicine, US Report Calls for Improved Suicide Research. 2002, MEDLINEplus, Washington.

21. Associate Minister of Health (2006) The New Zealand Suicide Prevention Strategy 2006-2016. Wellington: Ministry of Health.

22. Hakko H, Rasanen P, Tiihonen J, Nieminen P (2002) Use of statistical techniques in studies of suicide seasonality, 1970 to 1997 . Suicide Life Threat Behav 32: 191-208.

23. Shahtahmasebi S (2008) Can suicide be quantified and categorised? In Internet and Suicide L. Sher, Ed. Nova Sci: New York, 373-390.

24. Jordan JR (2001) Is suicide bereavement different? A reassessment of the literature. Suicide and Life-Threatening Behavior 31: 91-102.

25. Knieper AJ (1999) The suicide survivor's grief and recovery. Suicide and LifeThreatening Behavior 29: 353-364.

26. Constantino RE, Sekula LK, Rubinstein EN (2001) Group intervention for widowed survivors of suicide. Suicide and Life-Threatening Behavior 31: 428441.

27. Dyregrov K (2002) Assistance from local authorities versus survivors'needs for support after suicide. Death Studies 26: 647-668.

28. Jordan JR, McMenamy J (2004) Interventions for suicide survivors: A review of the literature. Suicide and Life-Threatening Behavior 34: 337-349.

29. Hung N, Rabin L (2009) Comprehending childhood bereavement by parental suicide: a critical review of research on outcomes, grief processes, and interventions. Death Stud 33: 781-814.

30. McMenamy J, Jordan J, Mitchell A (2008) What do suicide survivors tell us they need? Results of a pilot study. Suicide Life Threat Behav 38: 375-389.

31. Kubler-Ross E (1991) On Life after Death. Celestial Arts, USA

32. Lindsey AM, Yates BC (2004) Social support: conceptualization and measurement instruments. In Instruments for clinical health-care research 164 199.

33. Seible R (2001) Survivors of suicide victims searching for answers and ways out of the crisis. Psychiatr Prax 28: 348-350.

34. Shahtahmasebi S (2006) The Good life: A holistic approach to the health of the population. ScientificWorldJournal 6: 2117-2132.

35. Larson K (2007) Postvention strategies that will support a high school community through the stages of grief following a suicide event: A national Delphi study, University of la verne.

36. Omar HA (2005) A model program for youth suicide prevention. Int J Adolesc Med Health 17: 275-278.

37. Ehrhardt H, Ehrhardt P (2004) Support for Maori, Pacific and Asian family, whanau and significant others who have been bereaved by suicide. Ministry of Youth Development, Wellington.

38. Mitchell AM, Kim Y, Prigerson HG, Mortimer MK (2005) Complicated grief and suicidal ideation in adult survivors of suicide. Suicide and Life-Threatening Behavior 35: 498-506.

39. Fonagy P (1994) The theory and practice of resilience. J Child Psychol and Psychiatry 35: 231-257.

40. McMurray I, Connolly H, Preston-Shoot M, Wigley V (2008) Constructing resilience: Social workers' understandings and practice. Health and Socia Care in the Community 16: 299-309.

41. Pfeffer CR, Martins P, Mann J, Sunkenberg M, Ice A, et al. (1997) Child survivors of suicide: Psychosocial characteristics. Journal of the American Academy of Child \& Adolescent Psychiatry 36: 65-74.

42. Schneider B, Grebner K, Schnabel A, Georgi K (2011) Do suicides' characteristics influence survivors' emotions? Suicide \& Life-Threat Behav 41 117-125.

43. Ness DE, Pfeffer CR (1990) Sequelae of bereavement resulting from sucide. Americal Journal of Psychology 147: 279-285. 